\title{
Luminosity and abundance correlations in a carefully-studied sample of PNe
}

\author{
Jean McKeever ${ }^{1}$, Bruce Balick ${ }^{1}$, Julie Lutz ${ }^{1}$, Kelsey Braxton ${ }^{1}$,
} Thomas Gomez $^{1}$, Karen Kwitter ${ }^{2}$ and Richard C.B. Henry ${ }^{3}$

${ }^{1}$ Dept. of Astronomy, University of Washington,

Box 351580, Seattle WA 98195 USA

email: jeanm12@nmsu.edu, balick@uw.edu, jlutz@uw.edu, kmb537@uw.edu, gomezt@uw.edu

${ }^{2}$ Astronomy Department, Williams College,

33 Lab Campus Drive, Williamstown, MA 01267 USA

email: kkwitter@williams.edu

${ }^{3}$ H. L. Dodge Department of Physics and Astronomy, University of Oklahoma,

Nielsen Hall, Norman, OK 73019 USA

email: henry@nhn.ou .edu

\begin{abstract}
We have searched for correlated trends in $\mathrm{He} / \mathrm{H}, \mathrm{N} / \mathrm{H}, \mathrm{O} / \mathrm{H}, \mathrm{Ne} / \mathrm{H}, \mathrm{Ar} / \mathrm{H}, \mathrm{Cl} / \mathrm{H}$, and $\mathrm{S} / \mathrm{H}$ abundances with their luminosities, Peimbert types, galactocentric radius, emission-line luminosities, diameters, morphologies, surface brightnesses among Galactic planetary nebulae $(\mathrm{PNe})$ using a database of $119 \mathrm{PNe}$ with well measured properties. We report selected highlights.
\end{abstract}

Keywords. planetary nebulae: general, ISM: abundances, Galaxy: abundances

Using a sample of 119 well-measured Galactic planetary nebulae, we have searched for patterns that might reveal relationships and dependences of abundances with other measured properties among planetary nebulae $(\mathrm{PNe})$. For this we extracted a dataset of densities, temperatures, sizes, Peimbert types, morphologies, galactic locations, diameters, $\mathrm{H} \beta$ surface brightnesses, $\mathrm{H} \alpha$ and [OIII] luminosities, and $\mathrm{He}, \mathrm{C}, \mathrm{N}, \mathrm{O}, \mathrm{Ne}, \mathrm{Ar}$, and $\mathrm{S}$ abundances from a master compilation of high-quality observations described by Milingo et al. (2010). (A dozen PNe were rejected for their large and uncertain ionization correction factors.) Abundances are uniformly based on the ELSA abundance method of Johnson et al. (2006). [OIII] luminosities and surface brightnesses are based on extinction-corrected $\mathrm{H} \beta$ fluxes, angular sizes, and distances mostly from Cahn et al. (1992). All other data such as $[\mathrm{OIII}] 5007 / \mathrm{H} \beta$ ratios are computed from the entries in the database, including any necessary corrections for extinction and reddening.

Correlations in nebular abundances using $\mathrm{O} / \mathrm{H}$ as the independent variable. First we fitted the slopes of $12+\log (\mathrm{X} / \mathrm{H})$ vs. $12+\log (\mathrm{O} / \mathrm{H})$ where $(\mathrm{X}=\mathrm{He}, \mathrm{N}, \mathrm{Ne}, \mathrm{S}, \mathrm{Cl}$, and $\mathrm{Ar}$ ). The slopes for $\mathrm{Ne}, \mathrm{S}, \mathrm{Cl}$, and $\mathrm{Ar}$ are consistent with unity, suggesting that these elements are produced by common processes. The slope for $\mathrm{N} / \mathrm{H}$ is $0.75 \pm 0.11$. However, as a subgroup, Peimbert type-II PNe (PNe-II) have a slope of unity. PNe-I have a much flatter slope (not unexpected). We call this "Peimbert slope bifurcation", or "PSB." Similarly, the slopes for $\mathrm{He} / \mathrm{H}$ bifurcate with Peimbert type. The same pattern may apply also to $\mathrm{Cl} / \mathrm{H}$ and $\mathrm{Ar} / \mathrm{H}$; however, its significance is marginal.

Next we compared the same correlations within the same data set with another pair of groupings: (1) all 119 PNe, and (2) the 30 most luminous PNe in [OIII]. The 30 luminous PNe turn out to be a mixture of PNe-Is and PNe-IIs. We repeated the plots of various elements vs. $12+\log (\mathrm{O} / \mathrm{H})$. The slopes of the two samples agree well for $12+\log (\mathrm{Ne} / \mathrm{H})$. This confirms earlier findings that the $\mathrm{Ne} / \mathrm{O}$ ratio is universal. However, when compared 
to the full sample, the slopes of the luminous subsample are $\approx 10 \%$ larger than for the full sample in $\mathrm{He} / \mathrm{H}, \mathrm{S} / \mathrm{H}, \mathrm{Cl} / \mathrm{H}$, and $\mathrm{Ar} / \mathrm{H}$. As it turns out, these slopes agree surprisingly well with those of the subsample of the PNe-II. Differences in the slopes of $12+(\mathrm{N} / \mathrm{H})$ vs $12+\log (\mathrm{O} / \mathrm{H})$ cannot be clearly discerned due to scatter in the $\mathrm{N} / \mathrm{H}$ abundances.

Correlations in nebular abundances using $R_{\text {gal }}$ as the independent variable. Linear gradients are found in the logarithmic abundances of $\mathrm{N} / \mathrm{H}, \mathrm{O} / \mathrm{H}, \mathrm{Ne} / \mathrm{H}, \mathrm{S} / \mathrm{H}, \mathrm{Cl} / \mathrm{H}$, and $\mathrm{Ar} / \mathrm{H}$ with galactocentric radius, $R_{\text {gal }}$. These slopes are $-0.04,-0.03,-0.03,-0.02,-0.04,-$ 0.04 , and -0.07 , respectively with a scatter of $\sim 0.01-0.02$. He/H shows no gradient within the scatter. The 30 most luminous PNe follow the same trends as the entire sample, albeit with less scatter in some cases. The slopes are unchanged when the data sample is split into groups of PNe-I and PNe-II (as found by Henry et al. (2010). For He/H and N/H, PNe-I abundances are greater than those of PNe-II, as expected. Although the slope (i.e., gradient) uncertainties for each element are of order $50 \%$ (and more for $\mathrm{N} / \mathrm{H}$ ), the general tendency for the slope to steepen with atomic number appears to be real.

Correlations in observed line ratios using $\log \left(L_{\mathrm{H} \beta}\right)$ as the independent variable. We investigated whether the nebular excitation or ionization state varies with $\mathrm{H} \beta$ luminosity, $L_{\mathrm{H} \beta} . L_{\mathrm{H} \beta}$ was used as the independent variable since it simply measures the rate at which all ionizing photons are absorbed within the nebula, unlike $L_{[\mathrm{OIII}]}$. Various line ratios will reflect the evolution of stellar ionizing photon flux $\left(Q_{\mathrm{H}^{+}}\right)$, temperature $\left(T_{*}\right)$, and the change from ionization- to density-bounded structure. We use the line ratios $[\mathrm{NII}] \lambda 6584 / \mathrm{H} \alpha$ and $\mathrm{HeII} \lambda 4686 / \mathrm{H} \beta$ lines since these ratios are good indicators of the overall ionization state of the gas and they not sensitive to extinction.

The $[\mathrm{NII}] \lambda 6584 / \mathrm{H} \alpha$ ratio is systematically higher for PNe-I than PNe-II, as expected. Aside from this, the line ratio drops with increasing $L_{\mathrm{H} \beta}$. This is especially obvious for PNe-II since the ionization front (where $N^{+}$is brightest) exits the nebula as $T_{*}$ and $Q_{\mathrm{H}^{+}}$ increase with time. On the other hand PNe-Is tend to be bipolar, and their lobe edges are usually defined by persistent and extended ionization fronts.

The behavior of HeII $\lambda 4686 / \mathrm{H} \beta$ with $L_{\mathrm{H} \beta}$ is somewhat more surprising. The values of the line ratio lie between $0.1 \leqslant \operatorname{HeII} \lambda 4686 / \mathrm{H} \beta \leqslant 1$ with little dependence on $L_{\mathrm{H} \beta}$ or Peimbert type. However, smallest values of $\mathrm{HeII} / \mathrm{H} \beta-$ those with $\mathrm{HeII} / \mathrm{H} \beta \leqslant 0.1-$ are associated exclusively with the subsample of $\mathrm{PNe}$ with high $\mathrm{H} \beta$ luminosity; i.e., $\log \left(L_{\mathrm{H} \beta}\right)$ $\geqslant 34 \mathrm{erg} \mathrm{s}^{-1} \mathrm{~cm}^{-2}$. These turn out to be the same PNe for which $[\mathrm{NII}] / \mathrm{H} \alpha$ is smallest.

Finally, we searched for correlations of the nebular physical radius, $r_{\mathrm{PN}}$, with $L_{\mathrm{H} \beta}$. A very clear trend emerges: $\log \left(L_{\mathrm{H} \beta}\right)$ increases as $r_{\mathrm{PN}}$ increases from 0.02 to $0.05 \mathrm{pc}$; $\log \left(L_{\mathrm{H} \beta}\right)$ reaches its maximum value $(\sim 34.5)$ for $r_{\mathrm{PN}}$ between 0.05 and $0.1 \mathrm{pc}$. As $r_{\mathrm{PN}}$ continues to increase the corresponding values of $\log \left(L_{\mathrm{H} \beta}\right)$ drop precipitously. At $r_{\mathrm{PN}}$ $\sim 0.3 \mathrm{pc}, \log \left(L_{\mathrm{H} \beta}\right)$ has fallen to $\sim 33$ where, to all intents and purposes, it becomes difficult to measure. Thus the peak of the $\mathrm{H} \beta$ luminosity function corresponds to a narrow range of $\mathrm{PN}$ radii between 0.05 and $0.1 \mathrm{pc}$. We also find a discernible trend in $[\mathrm{NII}] \lambda 6584 / \mathrm{H} \alpha$ with $r_{\mathrm{PN}}$ and $L_{\mathrm{H} \beta}$. The line ratio attains a relative minimum $(\sim 0.1$ with some scatter $)$ at the peak of the $\mathrm{H} \beta$ luminosity function (i.e., $0.05<r_{\mathrm{PN}}<0.1$ ). The same results pertain equally to both PNe-Is and PNe-IIs.

\section{References}

Cahn, J. H., Kaler, J. B., \& Stanghellini, L. 1992, A\&AS, 94, 399

Henry, R. B. C., Kwitter, K. B., Jaskot, A. E., Balick, B., Morrison, M. A., \& Milingo, J. B. 2010, ApJ, 724, 748

Johnson, M. D., Levitt, J. S., Henry, R. B. C., \& Kwitter, K. B. 2006, Planetary Nebulae in our Galaxy and Beyond, 234, 439

Milingo, J. B., Kwitter, K. B., Henry, R. B. C., \& Souza, S. P. 2010, ApJ, 711, 619 